\title{
Quadrature Nodes Meet Stippling Dots
}

\author{
Manuel Gräf ${ }^{1}$, Daniel Potts ${ }^{1}$, and Gabriele Steidl ${ }^{2}$ \\ 1 Faculty of Mathematics, Chemnitz University of Technology, Germany \\ \{m.graef,potts\}@mathematik.tu-chemnitz.de \\ http://www.tu-chemnitz.de/ $\{$ grman,potts $\} /$ \\ 2 Department of Mathematics, University of Kaiserslautern, Germany \\ steidl@mathematik. uni-kaiserslautern.de \\ http://www.mathematik. uni-kl.de/ steidl/
}

\begin{abstract}
The stippling technique places black dots such that their density gives the impression of tone. This is the first paper that relates the distribution of stippling dots to the classical mathematical question of finding 'optimal' nodes for quadrature rules. More precisely, we consider quadrature error functionals on reproducing kernel Hilbert spaces (RKHSs) with respect to the quadrature nodes and suggest to use optimal distributions of these nodes as stippling dot positions. Interestingly, in special cases, our quadrature errors coincide with discrepancy functionals and with recently proposed attraction-repulsion functionals. Our framework enables us to consider point distributions not only in $\mathbb{R}^{2}$ but also on the torus $\mathbb{T}^{2}$ and the sphere $\mathbb{S}^{2}$. For a large number of dots the computation of their distribution is a serious challenge and requires fast algorithms. To this end, we work in RKHSs of bandlimited functions, where the quadrature error can be replaced by a least squares functional. We apply a nonlinear conjugate gradient (CG) method on manifolds to compute a minimizer of this functional and show that each step can be efficiently realized by nonequispaced fast Fourier transforms. We present numerical stippling results on $\mathbb{S}^{2}$.
\end{abstract}

\section{Introduction}

The traditionally artistic stippling technique places black dots to approximate different tones. For an illustration see Fig. 1. Stippling is closely related to dithering, where the dots have to lie on the image grid, see, e.g. [17] and the references therein. A popular stippling method proposed in [18] is based on weighted centroidal Voronoi tessellations and Lloyd's iterative algorithm $[13,5]$. A capacity-constrained variant of Lloyd's algorithm was introduced in [3]. Recently, a novel stippling approach was proposed in [21]: Consider a gray-value image $u: \mathcal{G} \rightarrow[0,1]$ on a grid $\mathcal{G}:=\frac{1}{n}(\mathbb{Z} / n \mathbb{Z}) \times \frac{1}{m}(\mathbb{Z} / m \mathbb{Z})$. Since 'black' is 0 and 'white' 1 , we use the weight $w:=1-u$. Now one intends to find the positions $p_{i} \in[0,1]^{2}, i=1, \ldots, M$, of $M$ black dots by minimizing the attraction-repulsion functional

$$
E(\boldsymbol{p}):=\sum_{i=1}^{M} \sum_{x \in \mathcal{G}} w(x)\left\|p_{i}-x\right\|_{2}-\frac{\lambda}{2} \sum_{i, j=1}^{M}\left\|p_{i}-p_{j}\right\|_{2}, \quad \boldsymbol{p}:=\left(p_{i}\right)_{i=1}^{M} \in \mathbb{R}^{2 M} .
$$


Here $\lambda:=\frac{1}{M} \sum_{x \in \mathcal{G}} w(x)$ is an equilibration parameter between the first sum which describes attracting forces caused by the image gray values and the second one which enforces repulsion between dots. The original idea for considering
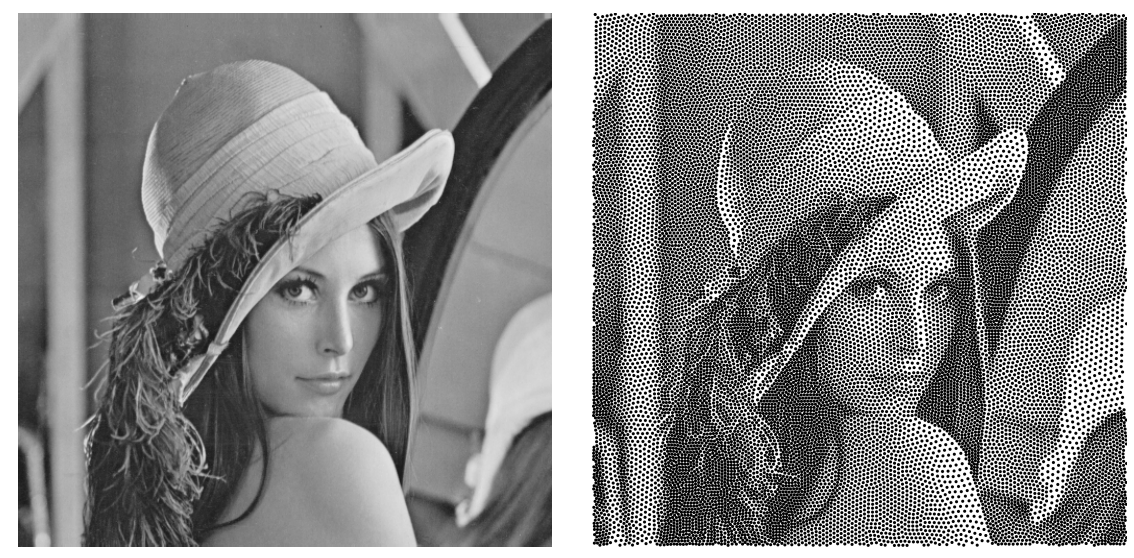

Fig. 1. Left: Original image. Right: Stippling result on $\mathbb{T}^{2}$ with $M=20000$ dots.

minimizers of this functional as 'good' dot positions comes from electrostatic principles used in [17]. This paper is related to the continuous version of the above attraction-repulsion functional with more general functions $\varphi:[0, \infty) \rightarrow \mathbb{R}$ :

$$
E_{\varphi}(\boldsymbol{p}):=\frac{\lambda}{2} \sum_{i, j=1}^{M} \varphi\left(\left\|p_{i}-p_{j}\right\|_{2}\right)-\sum_{i=1}^{M} \int_{[0,1]^{2}} w(x) \varphi\left(\left\|p_{i}-x\right\|_{2}\right) \mathrm{d} x .
$$

where $w:[0,1]^{2} \rightarrow[0,1]$ and $\lambda:=\frac{1}{M} \int_{[0,1]^{2}} w(x) \mathrm{d} x$. The function $\varphi(r)=-r$ was used in (1) and $\varphi(r)=-\log (r)$ in [17]. In [21] the authors mentioned $\varphi(r)=-r^{\tau}, 0<\tau<2$ and $\varphi(r)=r^{-\tau}, \tau>0$ for $r \neq 0$.

Contribution. In this paper we relate stippling processes with the classical mathematical question of finding best nodes for quadrature rules. We provide theoretical results on the connection between seemingly different concepts, namely quadrature rules, attraction-repulsion functionals, $L_{2}$-discrepancies and least squares functionals. For the later approach we provide numerical minimization algorithms.

In the theoretical part, we start with worst case quadrature errors on RKHSs in dependence on the quadrature nodes. While in the literature, this was mainly done for constant weights $w \equiv 1$, see $[10,15]$, we incorporate a weight function related to the image into the quadrature functional. The corresponding quadrature error $\operatorname{err}_{K}(\boldsymbol{p})$ which depends on the reproducing kernel $K$ can be defined for RKHSs on $\mathcal{X} \in\left\{\mathbb{R}^{2},[0,1]^{2}\right\}$ as well as for RKHSs on compact manifolds 
like $\mathcal{X} \in\left\{\mathbb{T}^{2}, \mathbb{S}^{2}\right\}$. We aim to minimize this quadrature error in order to obtain optimal quadrature nodes $\boldsymbol{p}$. It turns out that for special kernels $K$ (on special spaces $\mathcal{X}$ ) this quadrature error (or at least its minimizers) covers the following approaches:

1. Attraction-Repulsion Functionals

An interesting case of RKHSs appears for radial kernels $K(x, y)=\varphi(\| x-$ $y \|_{2}$ ) depending only on the distance of the points. We will show that in this case the quadrature error $\operatorname{err}_{K}(\boldsymbol{p})$ can be considered as a generalization of (2) which works not only on $[0,1]^{2}$ but also to compact manifolds. Hence our approach goes far beyond the setting in [21] or [17]. To get the special functional (1) from our general quadrature error we must stress conditionally positive definite, radial kernels of order 1.

2. $L_{2}$-Discrepancies

We prove that for $\mathcal{X} \in\left\{[0,1]^{2}, \mathbb{T}^{2}, \mathbb{S}^{2}\right\}$ and discrepancy kernels $K$, the quadrature errors on RKHSs defined by these kernels coincide with $L_{2}{ }^{-}$ discrepancy functionals. For various applications of $L_{2}$-discrepancy functionals, see $[15]$ and the references therein. Interestingly, this is also related to 'capacity constraints' in stippling techniques, see $[2,3]$. Note that a relation between the distance kernels $K(x, y)=\|x-y\|_{2}$ on $\mathbb{T}^{2}$ and $\mathbb{S}^{2}$ and the corresponding discrepancy kernels was shown numerically in [9].

3. Least Squares Functionals

Finally, we consider RKHSs of bandlimited functions with bandlimited kernels on $\mathcal{X} \in\left\{\mathbb{T}^{2}, \mathbb{S}^{2}\right\}$. The reason for addressing these spaces is that we want to approximate functions on $\mathcal{X}$ by bandlimited functions in order to apply fast Fourier techniques. We prove that for these RKHSs the quadrature error can be rewritten as a least squares functional.

In the numerical part we approximate functions and kernels on $\mathcal{X} \in\left\{\mathbb{T}^{2}, \mathbb{S}^{2}\right\}$ by their bandlimited versions and minimize the corresponding quadrature error which takes in this case the form of a least squares functional. Due to the page limitation we restrict our attention to the sphere $\mathbb{S}^{2}$. We are not aware of any results on $\mathbb{S}^{2}$-stippling in the literature. Note that a stippling example on the torus $\mathbb{T}^{2}$ was given in Fig. 1. We propose a nonlinear CG method on manifolds to compute a minimizer of the least squares functional on $\mathbb{S}^{2}$. This method was also successfully used for the approximation of spherical designs, i.e., for $w \sim 1$ in [8] and is generalized in this paper. In particular, each CG step can be realized in an efficient way by the nonequispaced fast spherical Fourier transform (NFSFT). This reduces the asymptotic complexity of the proposed algorithm drastically, e.g., from $\mathcal{O}\left(M N^{2}\right)$ to $\mathcal{O}\left(N^{2} \log ^{2} N+M \log ^{2}(1 / \epsilon)\right)$ arithmetic operations per iteration step, where $\epsilon$ is the described accuracy and $N$ corresponds to the bandwidth. In other words, only by the help of the NFSFT the computation becomes possible in a reasonable time.

Organization of the paper. In Sect. 2 we introduce our quadrature framework in RKHSs and show the relation to the attraction-repulsion functional. Sect. 3 relates this approach to discrepancy functionals. Sect. 4 deals with band-limited 
functions on $\mathbb{S}^{1}, \mathbb{T}^{2}$ and $\mathbb{S}^{2}$. We show that in this case the quadrature error can be written as a least squares functional. Moreover, we address the topic that bandlimited functions can be evaluated at a point set in a fast way by using the nonequispaced fast (spherical) Fourier transform. The same holds true for the evaluation of the functional itself. In Sect. 5, we propose a minimizing procedure on $\mathbb{S}^{2}$ by the CG method, where each iteration step can be efficiently computed by the NFSFT. Finally, Sect. 6 shows stippling results on $\mathbb{S}^{2}$ and Sect. 7 concludes the paper. Due to the page limitation proofs of certain theorems of this paper are given in the preprint [9].

\section{Quadrature Errors in RKHSs}

Let $\mathcal{X} \in\left\{\mathbb{R}^{2},[0,1]^{2}, \mathbb{T}^{2}, \mathbb{S}^{2}\right\}$. For notational reasons, we restrict our attention to two dimensions although the results are also true for arbitrary dimensions. A symmetric function $K: \mathcal{X} \times \mathcal{X} \rightarrow \mathbb{R}$ is said to be positive semi-definite if for any $M \in \mathbb{N}$ points $x_{1}, \ldots, x_{M} \in \mathcal{X}$ and any $a=\left(a_{1}, \ldots, a_{M}\right)^{\mathrm{T}} \neq 0$ the relation $a^{\mathrm{T}}\left(K\left(x_{i}, x_{j}\right)\right)_{i, j=1}^{M} a \geq 0$ holds true and positive definite if we have strict inequality. A (real) reproducing kernel Hilbert space (RKHS) is a Hilbert space having a reproducing kernel, i.e., a function $K: \mathcal{X} \times \mathcal{X} \rightarrow \mathbb{R}$ which fulfills

$$
K_{x}:=K(\cdot, x) \in H_{K} \quad \text { and } \quad f(x)=\langle f, K(\cdot, x)\rangle_{H_{K}}, \quad \forall x \in \mathcal{X}, \forall f \in H_{K} .
$$

To every RKHS there corresponds a unique positive semi-definite kernel and conversely given a positive semi-definite function $K$ there exists a unique RKHS of real-valued functions having $K$ as its reproducing kernel. By $\|\cdot\|_{H_{K}}$ we denote the norm of $H_{K}$. For more information on RKHSs we refer to [1].

In the following, let $w: \mathcal{X} \rightarrow \mathbb{R}_{\geq 0}$ be a nontrivial, continuous function which fulfills $h_{w}(x):=\int_{\mathcal{X}} w(y) K(x, y) \mathrm{d} y \in H_{K}$. We are interested in approximating

$$
I_{w}(f):=\int_{\mathcal{X}} f(x) w(x) \mathrm{d} x \quad \text { for } f \in H_{K}
$$

by a quadrature rule

$$
Q(f, \boldsymbol{p}):=\lambda \sum_{i=1}^{M} f\left(p_{i}\right), \quad \lambda:=\frac{1}{M} \int_{\mathcal{X}} w(x) \mathrm{d} x
$$

for appropriately chosen points $p_{j} \in \mathcal{X}$. In the literature mainly the case $w \equiv 1$ was considered, see $[10,15]$ and the references therein. In this paper, we have incorporated an image related weight $w$ into the functional. The worst case quadrature error is given by

$$
\operatorname{err}_{K}(\boldsymbol{p}):=\sup _{\substack{f \in H_{K} \\\|f\|_{H_{K}} \leq 1}}\left|I_{w}(f)-Q(f, \boldsymbol{p})\right|=\left\|I_{w}-Q(\cdot, \boldsymbol{p})\right\|_{H_{K}^{*}} .
$$

In the following we will see that this quadrature error covers various known functionals if we choose the kernel in an appropriate way. To start with, the following theorem shows a relation between the quadrature error functional and the attraction-repulsion functional (2). For a proof we refer to [9]. 
Theorem 1. (Quadrature Error and Attraction-Repulsion Functional) Let $K$ be a positive semi-definite function and $H_{K}$ the associated RKHS. Then the relation

$$
\operatorname{err}_{K}(\boldsymbol{p})^{2}=2 \lambda E_{K}(\boldsymbol{p})+\left\|h_{w}\right\|_{H_{K}}^{2}
$$

holds true, where

$$
E_{K}(\boldsymbol{p}):=\frac{\lambda}{2} \sum_{i, j=1}^{M} K\left(p_{i}, p_{j}\right)-\sum_{i=1}^{M} \int_{\mathcal{X}} w(x) K\left(p_{i}, x\right) \mathrm{d} x .
$$

In particular, the minimizers of $\operatorname{err}_{K}$ and $E_{K}$ coincide.

We see that for radial kernels $K(x, y):=\varphi\left(\|x-y\|_{2}\right)$ with some function $\varphi$ : $[0, \infty) \rightarrow \mathbb{R}$, the functional (4) has the form of an attraction-repulsion functional, where the first sum steers the repulsion of the dots and the second one the attraction. However, we have to ensure that the kernel is positive semi-definite. Positive definite, radial kernels on $\mathbb{R}^{2}$ are for example the inverse multiquadrics $K(x, y):=\left(\varepsilon^{2}+\|x-y\|_{2}^{2}\right)^{-\tau}, \varepsilon>0, \tau>1$ related to $\varphi(r):=r^{-\tau}$ in (2). These kernels and other positive semi-definite kernels do not lead to the functional (1).

Nevertheless, in the rest of this section, we will see how the attractionrepulsion functional in (1) fits into our quadrature setting. Of course choosing $\mathcal{X}:=\mathbb{R}^{2}$ and the radial kernel $K(x, y):=-\|x-y\|_{2}$ yields exactly (1). Unfortunately, this kernel is not positive semi-definite. However, it is conditionally positive definite of order 1 . Recall that a radial function $\Phi(x):=\varphi\left(\|x\|_{2}\right)$ is conditionally positive definite of order 1 if for any $M \in \mathbb{N}$ points $x_{1}, \ldots, x_{M} \in \mathbb{R}^{2}$ the relation

$$
a^{\mathrm{T}}\left(\Phi\left(x_{i}-x_{j}\right)\right)_{i, j=1}^{M} a>0 \quad \forall a=\left(a_{1}, \ldots, a_{M}\right)^{\mathrm{T}} \not \equiv 0 \quad \text { with } \quad \sum_{i=1}^{M} a_{i}=0 .
$$

holds true. Although these kernels are in general not positive semi-definite, the following slight modification of such kernels given by

$$
\tilde{K}_{\Phi}(x, y):=\Phi(x-y)-\Phi(y)-\Phi(x)+\Phi(0)+1
$$

defines again a positive semi-definite kernel $\tilde{K}_{\Phi}(x, y)$ which gives rise to a RKHS $H_{\tilde{K}_{\Phi}}$. The spaces $H_{\tilde{K}_{\Phi}}$ can be characterized as in [23]. Now it is not hard to show that the modification $\tilde{K}$ of a kernel $K(x, y)=\Phi(x-y)$ by (5) does not change the minimizer of the functional, i.e., then the minimizers of $E_{K}$ and $E_{\tilde{K}}$ coincide. In particular, we have for $K(x, y):=-\|x-y\|_{2}$ that $E_{K}$ and $E_{\tilde{K}}$ have the same minimizers, so that we can work with the original kernel $K$.

Finally, we mention with respect to various choices of $\varphi$ in (2) that other examples of conditionally positive definite, radial functions of order 1 are $\Phi(x):=$ $-\|x\|_{2}^{\tau}, 0<\tau<2$ and the multiquadrics $\Phi(x):=-\left(\varepsilon^{2}+\|x\|_{2}^{2}\right)^{\tau}, 0<\tau<1$. 


\section{Discrepancies}

The quadrature errors considered in the previous section are closely related to discrepancies which adds another interesting point of view. In the following we consider $\mathcal{X} \in\left\{[0,1]^{2}, \mathbb{S}^{1}, \mathbb{T}^{2}, \mathbb{S}^{2}\right\}$ as metric spaces with measure $\mu_{\mathcal{X}}$ and metric $\mathrm{d}_{\mathcal{X}}$. Let $D:=\mathcal{X} \times[0, R]$ and let $\mathcal{B}(c, r):=\left\{x \in \mathcal{X}: \mathrm{d}_{\mathcal{X}}(c, x) \leq r\right\}$ be the ball centered at $c \in \mathcal{X}$ with radius $0 \leq r \leq R$. By $1_{\mathcal{B}(c, r)}$ we denote the characteristic function of $\mathcal{B}(c, r)$. Then the kernel defined by

$$
K_{\mathcal{B}}(x, y):=\int_{0}^{R} \int_{\mathcal{X}} 1_{\mathcal{B}(c, r)}(x) 1_{\mathcal{B}(c, r)}(y) \mathrm{d} \mu_{\mathcal{X}}(c) \mathrm{d} r=\int_{0}^{R} \mu_{\mathcal{X}}(\mathcal{B}(x, r) \cap \mathcal{B}(y, r)) \mathrm{d} r
$$

is positive semi-definite. Consider for example the sphere $\mathbb{S}^{2}:=\left\{x \in \mathbb{R}^{3}\right.$ : $\left.\|x\|_{2}=1\right\}$ with the parameterization in spherical coordinates $x=x(\theta, \varphi):=$ $(\sin \theta \cos \varphi, \sin \theta \sin \varphi, \cos \theta)^{\mathrm{T}},(\varphi, \theta) \in[0,2 \pi) \times[0, \pi]$. The geodesic distance on $\mathbb{S}^{2}$ reads $\mathrm{d}_{\mathbb{S}^{2}}(x, y)=\arccos (x \cdot y)$ and the surface measure is given by $\mu_{\mathbb{S}^{2}}(x)=$ $\sin \theta \mathrm{d} \theta \mathrm{d} \varphi$. The balls are spherical caps and the area of the intersection of two caps $\mathcal{B}(c, r), 0 \leq r<\pi$ with center distance $d$ is

$$
a(r, d)= \begin{cases}0, & 0 \leq r \leq d / 2 \\ 4\left[\arccos \left(\sin \frac{d}{2} / \sin r\right)-\cos r \arccos \left(\tan \frac{d}{2} \cot r\right)\right], & \frac{d}{2}<r<\frac{\pi}{2} \\ 4 r-2 d, & r=\frac{\pi}{2}, \\ 4\left[\arccos \left(\sin \frac{d}{2} / \sin r\right)-\cos r \arccos \left(\tan \frac{d}{2} \cot r\right)\right], & \frac{\pi}{2}<r<\pi-\frac{d}{2} \\ -4 \pi \cos r, & \pi-\frac{d}{2} \leq r<\pi\end{cases}
$$

The corresponding discrepancy kernel is $K_{\mathcal{B}}(d)=\int_{0}^{\pi} a(r, d) \mathrm{d} r$. For examples of discrepancy kernels on $[0,1]^{d}, \mathbb{S}^{1}$ and $\mathbb{T}^{2}$ and their relations to distance kernels $\Phi(x, y)=-\|x-y\|_{2}$, we refer to $[15,9]$.

Integration on the RKHSs $H_{K_{\mathcal{B}}}$ is related to the notation of discrepancy. Set $t:=(c, r) \in D$ and $\mathrm{d} t:=\mathrm{d} \mu_{\mathcal{X}}(c) \mathrm{d} r$. We define the $L_{2}$-discrepancy as

$$
\operatorname{disc}_{2}^{\mathcal{B}}(\boldsymbol{p}):=\left(\int_{D}\left(\int_{\mathcal{X}} w(x) 1_{\mathcal{B}(t)}(x) d x-\lambda \sum_{i=1}^{M} 1_{\mathcal{B}(t)}\left(p_{i}\right)\right)^{2} \mathrm{~d} t\right)^{\frac{1}{2}}
$$

The expression in the inner brackets relates the integral of $w$ on $\mathcal{B}(c, r)$ with the number of points contained in $\mathcal{B}(c, r)$ for fixed $(c, r) \in D$. The discrepancy is then the squared error of their differences taken over all $t \in D$. This point of view is closely related to capacity-constrained methods used in $[2,3]$. The relation between the discrepancy and the quadrature error is given by the next theorem.

Theorem 2. (Quadrature Error and $L_{2}$-Discrepancy)

Let $K_{\mathcal{B}}$ be defined by (6) and let $H_{K_{\mathcal{B}}}$ be the associated RKHS of functions on $\mathcal{X}$. Then $\operatorname{err}_{K_{\mathcal{B}}}$ given by (3) and $\operatorname{disc}_{2}^{\mathcal{B}}$ determined by (7) coincide

$$
\operatorname{err}_{K_{\mathcal{B}}}(\boldsymbol{p})=\operatorname{disc}_{2}^{\mathcal{B}}(\boldsymbol{p})
$$




\section{Least Squares Functionals for Bandlimited Functions}

Let $\mathcal{X} \in\left\{\mathbb{T}^{2}, \mathbb{S}^{2}\right\}$ and let $\left\{\psi_{l}: l \in \mathbb{N}\right\}$ be an orthonormal basis of $L_{2}(\mathcal{X})$. Then any real-valued function $w \in L_{2}(\mathcal{X})$ can be written in the form

$$
w(x)=\sum_{l=1}^{\infty} \hat{w}_{l} \psi_{l}(x), \quad \hat{w}_{l}=\left\langle f, \psi_{l}\right\rangle_{L_{2}}=\int_{\mathcal{X}} w(x) \overline{\psi_{l}(x)} \mathrm{d} x .
$$

In order to develop fast algorithms for the efficient computation of minimizers $\hat{\boldsymbol{p}}$ of functionals $E_{K}$ we will work in spaces of bandlimited functions $\Pi_{N}(\mathcal{X}):=$ $\operatorname{span}\left\{\psi_{l}: l=1, \ldots, d_{N}\right\}$ of dimension $d_{N}:=\operatorname{dim} \Pi_{N}(\mathcal{X})$. More precisely, we will use the spaces $\Pi_{N}\left(\mathbb{S}^{1}\right):=\operatorname{span}\left\{\mathrm{e}^{-2 \pi \mathrm{i} n(\cdot)}: n=-N / 2, \ldots, N / 2\right\}, \Pi_{N}\left(\mathbb{T}^{2}\right):=$ $\operatorname{span}\left\{\mathrm{e}^{-2 \pi \mathrm{i} n(\cdot)}: n=\left(n_{1}, n_{2}\right), n_{j}=-N / 2, \ldots, N / 2, j=1,2\right\}$ with even $N$ and $\Pi_{N}\left(\mathbb{S}^{2}\right):=\operatorname{span}\left\{Y_{n}^{k}: n=0, \ldots, N ; k=-n, \ldots, n\right\}$. Here $Y_{n}^{k}$ denote the spherical harmonics of degree $n$ and order $k$, see [14]. We will apply bandlimited kernels of the form

$$
K_{N}(x, y):=\sum_{l=1}^{d_{N}} \lambda_{l} \psi_{l}(x) \overline{\psi_{l}(y)}
$$

with $\lambda_{l}>0$. These kernels are reproducing kernels for the RKHSs $H_{K_{N}}:=$ $\Pi_{N}(\mathcal{X})$ with the inner product $\langle f, g\rangle_{H_{K_{N}}}=\sum_{l=1}^{d_{N}} \hat{f}_{l} \overline{\hat{g}}_{l} / \lambda_{l}$. For the efficient computation of minimizers of $E_{K_{N}}$ it is useful to rewrite the functional in weighted least squares form.

Theorem 3. (Quadrature Error and Least Squares Functional)

Let the kernel $K_{N}$ be given by (8). Then the relation $\operatorname{err}_{K_{N}}(\boldsymbol{p})^{2}=\mathcal{E}_{N}(\boldsymbol{p})$ holds true, where

$$
\mathcal{E}_{N}(\boldsymbol{p}):=\sum_{l=1}^{d_{N}} \lambda_{l}\left|\lambda \sum_{i=1}^{M} \overline{\psi_{l}\left(p_{i}\right)}-\hat{w}_{l}\right|^{2}=\left\|\boldsymbol{\Lambda}^{\frac{1}{2}} F(\boldsymbol{p})\right\|_{2}^{2}
$$

with $\boldsymbol{\Lambda}:=\operatorname{diag}\left(\lambda_{l}\right)_{l=1}^{d_{N}}$ and $F(\boldsymbol{p})=\left(F_{l}(\boldsymbol{p})\right)_{l=1}^{d_{N}}, F_{l}(\boldsymbol{p}):=\lambda \sum_{i=1}^{M} \overline{\psi_{l}\left(p_{i}\right)}-\hat{w}_{l}$. In particular, the functionals $E_{K_{N}}$ and $\mathcal{E}_{N}$ have the same minimizers.

Note that for $\mathcal{X}=\mathbb{S}^{2}$ and $w \equiv 1$ there is a close relation between the minimizers $\mathcal{E}_{N}(\boldsymbol{p})$ and spherical designs, see [19,8,9].

The evaluation of bandlimited functions

$$
f\left(p_{i}\right)=\sum_{l=1}^{d_{N}} \hat{f}_{l} \psi_{l}\left(p_{i}\right), \quad i=1, \ldots, M
$$

on $\mathcal{X} \in\left\{\mathbb{S}^{1}, \mathbb{T}^{2}, \mathbb{S}^{2}\right\}$ can be written in matrix-vector form as $\boldsymbol{f}=\boldsymbol{A}_{N} \hat{\boldsymbol{f}}$, where $\boldsymbol{f}:=\left(f\left(p_{i}\right)\right)_{i=1}^{M}, \hat{\boldsymbol{f}}:=\left(\hat{f}_{l}\right)_{l=1}^{d_{N}}$ appropriately ordered and

$$
\boldsymbol{A}_{N}:= \begin{cases}\boldsymbol{F}_{N}=\left(\mathrm{e}^{-2 \pi \mathrm{i} n p_{i}}\right)_{i=1, \ldots, M ; n=-N / 2, \ldots, N / 2} & \text { for } \mathbb{S}^{1}, \\ \boldsymbol{F}_{2, N}=\left(\mathrm{e}^{-2 \pi \mathrm{i}\left(n_{1}, n_{2}\right)^{\mathrm{T}} \cdot p_{i}}\right)_{i=1, \ldots, M ; n_{i}=-N / 2, \ldots, N / 2, i=1,2} & \text { for } \mathbb{T}^{2}, \\ \boldsymbol{Y}_{N}=\left(Y_{k}^{n}\left(p_{i}\right)\right)_{i=1, \ldots, M ; n=0, \ldots, N,|k| \leq n} & \text { for } \mathbb{S}^{2} .\end{cases}
$$


The main reason for working in spaces of bandlimited function is the existence of fast algorithms for the matrix-vector multiplication with $\boldsymbol{A}_{N}$ and $\overline{\boldsymbol{A}}_{N}^{\mathrm{T}}$.

\section{Theorem 4. (Fast Evaluation of Bandlimited Functions)}

The nonequispaced fast Fourier transform (NFFT) and the nonequispaced fast spherical Fourier transform (NFSFT) realize the multiplication of a vector with the matrix $\boldsymbol{A}_{N}$, resp. $\overline{\boldsymbol{A}}_{N}^{T}$, with the following number of arithmetic operations: $\mathcal{O}(N \log N+M \log (1 / \epsilon))$ for $\mathbb{S}^{1}, \mathcal{O}\left(N^{2} \log N+M \log ^{2}(1 / \epsilon)\right)$ for $\mathbb{T}^{2}$ and $\mathcal{O}\left(N^{2} \log ^{2} N+M \log ^{2}(1 / \epsilon)\right)$ for $\mathbb{S}^{2}$, where $\epsilon$ is the prescribed accuracy.

For the NFFT we refer to $[6,4,16,12]$ and for the NFSFT to $[11,12]$. It can be shown that using these algorithms the same complexity is required for the evaluation of $\mathcal{E}_{N}(\boldsymbol{p})$.

\section{Efficient Minimization Algorithm on $\mathbb{S}^{2}$}

In this section, we describe the computation of a local minimizers of $\mathcal{E}_{N}$ in an efficient way for given $\hat{w}_{l}, l=1, \ldots, d_{N}$. We restrict our attention to the case $\mathcal{X}=\mathbb{S}^{2}$, where we will only work with kernels of the form

$$
K_{N}(x, y):=\sum_{n=0}^{N} \sum_{k=-n}^{n} \lambda_{n} Y_{n}^{k}(x) \overline{Y_{n}^{k}(y)} .
$$

such that

$$
\mathcal{E}_{N}(\boldsymbol{p})=\sum_{n=0}^{N} \sum_{k=-n}^{n} \lambda_{n}\left|\lambda \sum_{i=1}^{m} \overline{Y_{n}^{k}\left(p_{i}\right)}-\hat{w}_{n}^{k}\right|^{2} .
$$

Using the considerations of the previous section similar algorithms can be deduced for $\mathcal{X} \in\left\{\mathbb{S}^{1}, \mathbb{T}^{2}\right\}$.

Due to the good experiences in connection with spherical designs in [8] we apply the nonlinear $\mathrm{CG}$ method on the manifold $\mathcal{M}:=\left(\mathbb{S}^{2}\right)^{M}$, cf. [20].

Algorithm: (CG algorithm on Riemannian manifolds)

Initialization: $\boldsymbol{p}^{(0)}, \boldsymbol{h}^{(0)}:=\nabla \mathcal{E}_{N}\left(\boldsymbol{p}^{(0)}\right), \boldsymbol{d}^{(0)}=-\boldsymbol{h}^{(0)}$

For $r=0,1, \ldots$ repeat until a convergence criterion is reached

1. $\alpha_{r}:=-\left\langle\boldsymbol{d}^{(r)}, \boldsymbol{h}^{(r)}\right\rangle /\left\langle\boldsymbol{d}^{(r)}, \mathrm{H} \mathcal{E}_{N}\left(\boldsymbol{p}^{(r)}\right) \boldsymbol{d}^{(r)}\right\rangle$

2. $\boldsymbol{p}^{(r+1)}:=\exp _{\boldsymbol{p}^{(r)}}\left(\alpha_{r} \boldsymbol{d}^{(r)}\right)$

3. $\boldsymbol{h}^{(r+1)}:=\nabla \mathcal{E}_{N}\left(\boldsymbol{p}^{(r+1)}\right)$

4. Compute $\beta_{r}$ by $\quad \beta_{r}:=\frac{\left\langle\boldsymbol{h}^{(r+1)}, \mathrm{H} \mathcal{E}_{N}\left(\boldsymbol{p}^{(r+1)}\right) \tilde{\boldsymbol{d}}^{(r)}\right\rangle}{\left\langle\tilde{\boldsymbol{d}}^{(r)}, \mathrm{H} \mathcal{E}_{N}\left(\boldsymbol{p}^{(r+1)}\right) \tilde{\boldsymbol{d}}^{(r)}\right\rangle}, \quad \tilde{\boldsymbol{d}}^{(r)}:=\boldsymbol{P}_{\boldsymbol{g}\left(\alpha_{r}\right)}\left(\boldsymbol{d}^{(r)}\right)$.

5. $\boldsymbol{d}^{(r+1)}:=-\boldsymbol{h}^{(r+1)}+\beta_{r} \tilde{\boldsymbol{d}}^{(r)}$

Here $\exp _{\boldsymbol{p}}: \mathrm{T}_{\boldsymbol{p}} \mathcal{M} \rightarrow \mathcal{M}$ denotes the exponential map from the tangent space $\mathrm{T}_{\boldsymbol{p}} \mathcal{M}$ to the manifold and $\boldsymbol{P}_{\boldsymbol{g}\left(\alpha_{r}\right)}\left(\boldsymbol{d}^{(r)}\right)$ the parallel transport of $\boldsymbol{d}^{(r)} \in \mathrm{T}_{\boldsymbol{p}^{(r)}} \mathcal{M}$ along the geodesics $\boldsymbol{g}$. For the manifold notation including the concept of parallel transport we refer to [22]. 
Each CG step requires the evaluation of the gradient of $\mathcal{E}_{N}$ and the multiplication of the Hessian of $\mathcal{E}_{N}$ with a vector. By the following corollary both computations can be done in an efficient way.

Theorem 5. (Fast Evaluation of $\nabla \mathcal{E}_{N}$ and Multiplication with $\mathrm{H} \mathcal{E}_{N}$ ) For a given point $\boldsymbol{p} \in\left(\mathbb{S}^{2}\right)^{M}$ and given $\hat{w}_{n}^{k}$, the gradient $\nabla \mathcal{E}_{N}(\boldsymbol{p})$ can be evaluated with the arithmetic complexity $\mathcal{O}\left(N^{2} \log ^{2} N+M \log ^{2}(1 / \epsilon)\right)$. The multiplication of a vector with the Hessian $\mathrm{H}_{N}(\boldsymbol{p})$ can be computed with the same complexity.

For the proof we refer to the accompanying paper [9].

\section{$6 \quad$ Numerical results on $\mathbb{S}^{2}$}

In this section, we present some stippling results on $\mathbb{S}^{2}$. The proposed algorithms were implemented in Matlab R2010a, where the mex-interface to the NFFT library [12] was used. The internal library parameters were set as follows: cutoff parameter $m=9$, threshold parameter $\kappa=1000$, flags PRE_PSI and PRE_PHI_HUT. From the sampling points $\boldsymbol{x}:=\left(x_{i}\right)_{i=1}^{L} \in\left(\mathbb{S}^{2}\right)^{L}$ of the function $w$ we obtain approximate Fourier coefficients

$$
\hat{w}_{n}^{k}:=\sum_{i=1}^{L} \omega_{i} w\left(x_{i}\right) \overline{Y_{n}^{k}\left(x_{i}\right)}, \quad l=1, \ldots, d_{N},
$$

where the quadrature weights $\omega_{i}$ are chosen such that $\int_{\mathbb{S}^{2}} f(x) \mathrm{d} x=\sum_{i=1}^{L} \omega_{i} f\left(x_{i}\right)$ for all $f \in \Pi_{N}\left(\mathbb{S}^{2}\right)$. Note that the above sums can be evaluated in an efficient way by the NFSFT. As kernel we use the bandlimited version of the distance kernel $\Phi(x-y)=-\|x-y\|_{2}=-2 \sin \left(\mathrm{d}_{\mathbb{S}^{2}}(x, y) / 2\right), x, y \in \mathbb{S}^{2}$, where the coefficients in (9) are explicitly given by

$$
\lambda_{n}=\frac{16 \pi}{(2 n+3)(2 n+1)(2 n-1)}, \quad n \in \mathbb{N}_{0} .
$$

We apply the CG algorithm for randomly distributed initial points $\boldsymbol{p}^{(0)}$.

The first example uses the topography map of the earth from Matlab. This map consists of the earth's elevation data. Since the values ranging from -7473 to 5731 we have scaled them to $[0,1]$, in order to avoid negative values. The data is sampled on the grid $\boldsymbol{x}:=(x(\pi i / 180, \pi j / 180))_{i=1, j=1}^{180,360}$. For this grid we have computed nonnegative quadrature weights $\omega_{i, j}$ for a polynomial degree $N=179$ by the simple CG algorithm proposed in [7]. After applying the quadrature rule (10) we obtain a polynomial approximation $w=\sum_{n=0}^{179} \sum_{k=-n}^{n} \hat{w}_{n}^{k} Y_{n}^{k}$ of the earth's topography, see the left-hand side of Figure 2. For $M=200000$ and a kernel of bandwidth $N=1000$, our algorithm obtained after $r=3600$ iterations the right image in Figure 2, where one iteration takes about 1.5 minutes.

We remark that a naive evaluation of the attraction-repulsion functional (1) requires at least $\mathcal{O}\left(M^{2}\right)$ arithmetic operations. If we approximate the kernel $\Phi(x-y)=-\|x-y\|_{2}$ by bandlimited kernels $K_{N}$ of the form (9) with $N^{2} \sim M$, 
then every step in the proposed nonlinear CG method needs $\mathcal{O}\left(M \log ^{2} M+\right.$ $\left.M \log ^{2}(1 / \epsilon)\right)$ arithmetic operations. For a crude illustration of the performance gain in our implementation to a naive one we run our algorithm with the slow NDFST and fast NFSFT. Under the above assumption one iteration step with the NDFST needs also $\mathcal{O}\left(M^{2}\right)$ operations. In our examples, the algorithm takes per iteration about 1.5 minutes versus 3 hours with the NDFST. This reveals the importance of fast algorithms for huge numbers of points.
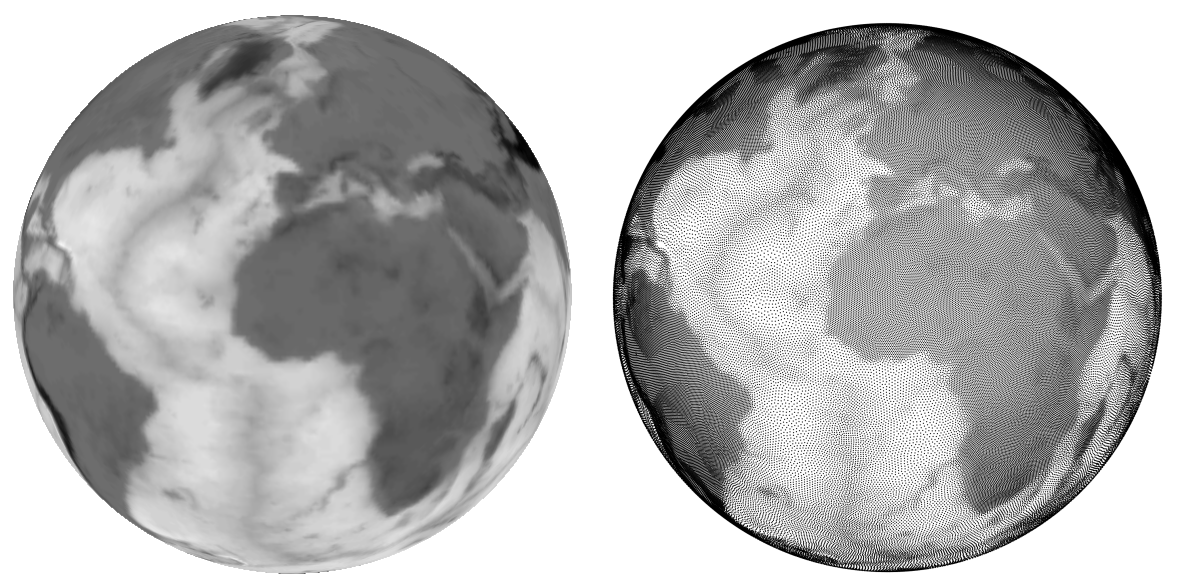

Fig. 2. Left: Original image. Right: Stippling result with $M=200000$ points.

In the second example we map a section of the left bitmap of Figure 1 on the sphere by the same grid as in the first example. The stippling result after $r=500$ iterations is presented in Figure 3, where we used $M=100000$ points and a bandwidth $N=1000$.

\section{Conclusions}

In this paper, we had a look at the stippling problem from different points of view. Our framework arises primarily from approximation theory but touches many different areas in mathematics as well. The proposed setting is quite general and enables us to consider in some sense 'optimal' point distributions not only in $\mathbb{R}^{2}$ but also on the torus $\mathbb{T}^{2}$ and the sphere $\mathbb{S}^{2}$. Note that even in the seemingly easiest case $w \equiv 1$ the search for optimal point configurations in more than one dimension is a very tough problem which originated many publications. In this case for translationally invariant kernels on $\mathbb{T}^{2}, \mathbb{S}^{2}$ the attraction term in (4) is constant and can be omitted.

We have clarified the relation of our quadrature error functionals to recently applied attraction-repulsion functionals and to $L_{2}$-discrepancy functionals. For 

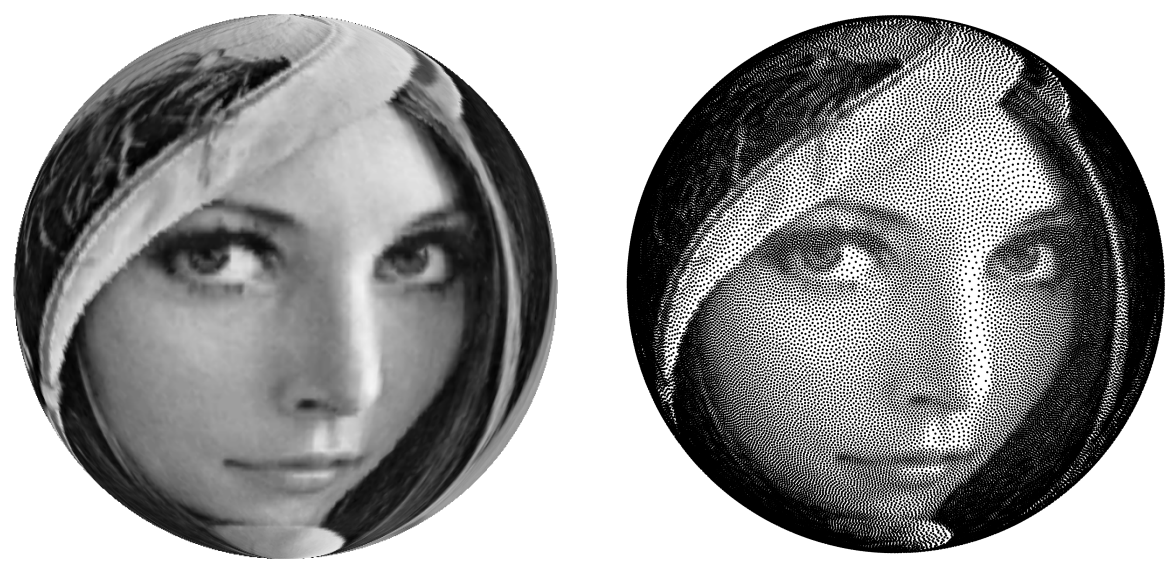

Fig. 3. Left: Original image. Right: Stippling result with $M=100000$ points.

bandlimited functions on $\mathbb{S}^{1}, \mathbb{T}^{2}$ and $\mathbb{S}^{2}$ we suggested to rewrite the quadrature error functional in a least squares form. This is summarized in the figure below. Then the nonlinear CG algorithm can be applied in conjunction with efficient NF(S)FTs for stippling on $\mathbb{S}^{2}$.

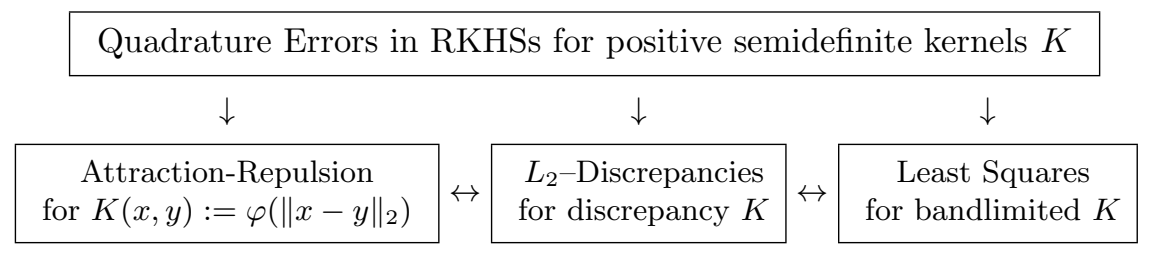

\section{References}

1. N. Aronszajn. Theory of reproducing kernels. Trans. Amer. Math. Soc., 68:337 404, 1950.

2. F. Aurenhammer, F. Hoffmann, and B. Aronov. Minkowski-type theorems and least-squares clustering. Algorithmica, 20:61 - 76, 1998.

3. M. Balzer, T. Schlömer, and O. Deussen. Capacity-constrained point distributions: A variant of Lloyd's method. ACM Transactions on Graphics, 28(3):Article 86, 2009.

4. G. Beylkin. On the fast Fourier transform of functions with singularities. Appl. Comput. Harmon. Anal., 2:363 - 381, 1995.

5. Q. Du, V. Faber, and M. Gunzburger. Centroidal Voronoi tessellations: Applications and algorithms. SIAM Review, 41:637 - 676, 1999.

6. A. Dutt and V. Rokhlin. Fast Fourier transforms for nonequispaced data. SIAM J. Sci. Stat. Comput., 14:1368 - 1393, 1993. 
7. M. Gräf, S. Kunis, and D. Potts. On the computation of nonnegative quadrature weights on the sphere. Appl. Comput. Harmon. Anal., 27:124-132, 2009.

8. M. Gräf and D. Potts. On the computation of spherical designs by a new optimization approach based on fast spherical Fourier transforms. TU Chemnitz, Fakultät für Mathematik, Preprint 10, 2010.

9. M. Gräf, D. Potts, and G. Steidl. Quadrature errors, discrepancies and their relations to halftoning on the torus and the sphere. TU Chemnitz, Fakultät für Mathematik, Preprint 5, 2011.

10. S. Graf and H. Luschgy. Foundations of Quantization for Probability Distributions. Springer, LNM 1730, Berlin - Heidelberg - New York, 2000.

11. D. M. Healy, P. J. Kostelec, and D. Rockmore. Towards Safe and Effective HighOrder Legendre Transforms with Applications to FFTs for the 2-sphere. $A d v$. Comput. Math., 21:59 - 105, 2004.

12. J. Keiner, S. Kunis, and D. Potts. Using NFFT3 - a software library for various nonequispaced fast Fourier transforms. ACM Trans. Math. Software, 36:Article 19, $1-30,2009$.

13. S. P. Lloyd. Least square quantization in PCM. IEEE Transactions on Information Theory, 28:129 - 137, 1982.

14. C. Müller. Spherical Harmonics. Springer, Aachen, 1966.

15. E. Novak and H. Woźniakowski. Tractability of Multivariate Problems Volume II: Standard Information for Functionals. Eur. Math. Society, EMS Tracts in Mathematics Vol 12, 2010.

16. D. Potts, G. Steidl, and M. Tasche. Fast Fourier transforms for nonequispaced data: A tutorial. In J. J. Benedetto and P. J. Ferreira, editors, Modern Sampling Theory: Mathematics and Applications, pages 247 - 270, Boston, MA, USA, 2001. Birkhäuser.

17. C. Schmaltz, P. Gwosdek, A. Bruhn, and J. Weickert. Electrostatic halftoning. Computer Graphics Forum, 29:2313 - 2327, 2010.

18. A. Secord. Weighted Voronoi stippling. In Proceedings of the 2nd International Symposium on Non-Photorealistic Animation and Rendering, pages 37 - 43. ACM Press, 2002.

19. I. H. Sloan and R. S. Womersley. A variational characterisation of spherical designs. J. Approx. Theory, 159:308 - 318, 2009.

20. S. T. Smith. Optimization techniques on Riemannian manifolds. In Hamiltonian and gradient flows, algorithms and control, volume 3 of Fields Inst. Commun., pages 113 - 136. Amer. Math. Soc., Providence, RI, 1994.

21. T. Teuber, G. Steidl, P. Gwosdek, Ch. Schmaltz, and J. Weickert. Dithering by differences of convex functions. SIAM J. Imaging Sci., accepted.

22. C. Udrişte. Convex functions and optimization methods on Riemannian manifolds, volume 297 of Mathematics and its Applications. Kluwer Academic Publishers Group, Dordrecht, 1994.

23. H. Wendland. Scattered Data Approximation. Cambridge University Press, Cambridge, 2005. 